\title{
Study protocol: older people in retirement villages. A survey and randomised trial of a multi-disciplinary invention designed to avoid adverse outcomes
}

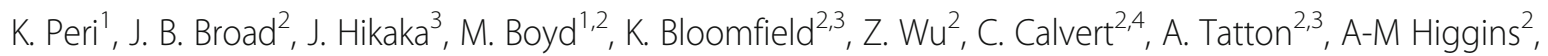

D. Bramley ${ }^{3}$ and M. J. Connolly ${ }^{2,3^{*}}$ (D)

\begin{abstract}
Background: There is increasing interest among older people in moving into retirement villages (RVs), an attractive option for those seeking a supportive community as they age, while still maintaining independence. Currently in New Zealand there is limited knowledge of the medical, service supports, social status and needs of RV residents. The objective of this study is to explore RV facilities and services, the health and functional status of RV residents, prospectively study their healthcare trajectories and to implement a multidisciplinary team intervention to potentially decrease dependency and impact healthcare utilization.

Methods: All RVs located in two large district health boards in Auckland, New Zealand were eligible to participate. This three-year project comprised three phases: The survey phase provided a description of RVs, residents' characteristics and health and functional status. RV managers completed a survey of size, facilities and recreational and healthcare services provided in the village. Residents were surveyed to establish reasons for entry to the village and underwent a Gerontology Nurse Specialist (GNS) assessment providing details of demographics, social engagement, health and functional status. The cohort study phase examines residents' healthcare trajectories and adverse outcomes, over three years. The final phase is a randomised controlled trial of a multidisciplinary team intervention aimed to improve health outcomes for more vulnerable residents.

Residents who triggered potential unmet health needs during the assessment in the survey phase were randomised to intervention or usual care groups. Multidisciplinary team meetings included the resident and support person, a geriatrician or gerontology nurse practitioner, GNS, pharmacist and General Practitioner. The primary outcome of the randomised controlled trial will be first acute hospitalization. Secondary outcomes include all acute hospitalizations, long-term care admissions, and all-cause mortality.
\end{abstract}

(Continued on next page)

\footnotetext{
*Correspondence: martin.connolly@waitematadhb.govt.nz

${ }^{2}$ Department of Geriatric Medicine, University of Auckland, Level 1, Building

5. Waitemata District Health Board, PO Box 93 503, Auckland, Takapuna 0740, New Zealand

${ }^{3}$ Waitemata District Health Board, PO Box 93 503, Auckland, Takapuna 0740, New Zealand

Full list of author information is available at the end of the article
}

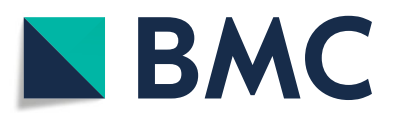

(- The Author(s). 2020 Open Access This article is licensed under a Creative Commons Attribution 4.0 International License, which permits use, sharing, adaptation, distribution and reproduction in any medium or format, as long as you give appropriate credit to the original author(s) and the source, provide a link to the Creative Commons licence, and indicate if changes were made. The images or other third party material in this article are included in the article's Creative Commons licence, unless indicated otherwise in a credit line to the material. If material is not included in the article's Creative Commons licence and your intended use is not permitted by statutory regulation or exceeds the permitted use, you will need to obtain permission directly from the copyright holder. To view a copy of this licence, visit http://creativecommons.org/licenses/by/4.0/. The Creative Commons Public Domain Dedication waiver (http://creativecommons.org/publicdomain/zero/1.0/) applies to the data made available in this article, unless otherwise stated in a credit line to the data. 
(Continued from previous page)

Discussion: This paper describes the study protocol of this complex study. The study aims to inform policies and practices around health care services for residents in retirement villages. The results of this trial are expected early 2020 with publication subsequently.

Trial registration: Australia and New Zealand Clinical Trials Registry: ACTRN12616000685415. Registered 25.5.2016. Universal Trial Number (UTN): U111-1173-6083.

Keywords: Older people, Retirement villages, Health status

\section{Background}

The retirement village (RV) industry provides an attractive housing option for older people who seek a supportive community while still maintaining independence as they age [1]. The term "Retirement Village" in New Zealand (NZ) is also known internationally as a Continuing Care Retirement Community (CCRC), defined as full range lifetime of care from independent to assisted living. The term RV is legally defined in accordance with NZ's Retirement Villages Act 2003 as "part of any property, building or other premises that contains two or more residential units that provide, or are intended to provide residential accommodation together with services or facilities or both, predominantly for persons in their retirement and their spouses or partners" [2].

There has been a significant growth in RVs in NZ as well as internationally in high resourced countries. From 1988 to 2008, the population of people over 85 years has more than doubled in Auckland, NZ [3]. In spite of this, long-term care (LTC) beds for those requiring 24-h care decreased by almost a third during this same time period, likely due to government policies that promoted community care and "ageing in place" [3-5]. This is likely to have resulted in a trend of increased physical dependency and healthcare complexity for those currently living in RVs $[6,7]$. During this same time period, the RV sector saw enormous growth. Recent estimates indicate that there are 380 registered RV complexes in NZ, with 390,000 occupants, a three-fold increase from 1998. Between 2010 and 2016, 40 new RVs opened - a growth rate of $12 \%$. There were almost no RV residents in NZ in the late 1980s, yet by 2017 an estimated 12.6\% of people of 75 years and over in NZ lived in RVs [8].

Aside from 'ageing in place' and the overall ageing of the population, there are a number of drivers responsible for the increase in demand for RV units (villas/apartments). RV living is growing in popularity with an increasing range of housing and lifestyle 'products' to choose from and providing an alternative to traditional lower level residential aged care. Other influences include NZ's recently strong local housing market and the relative affordability of RV living, and a decline in alternative accommodation options (such as the decline in ageing parents living with their children) [8].
Internationally, RVs have been shown to impact positively on residents' wellbeing, with higher quality of life compared to those living in family homes or in LTC $[9$, 10]. NZ research has shown that RV residents are more educated, have more financial resources and better wellbeing, but have significantly greater functional dependency compared with those in private dwellings [11]. It is clear that only those with substantial financial resources can afford the significant personal investment required initially (NZ\$250,000- NZ\$1 M+) and the (usually) ongoing monthly service fees, [8] resulting in inequity in access.

An increase in the ageing population in NZ is projected over the next decade. The number of older people living within RVs will also likely increase, who as they age will be more prone to increased frailty, greater health needs and declining independence. Indeed, it is likely that RV residents may include a proportion of frailer dependent older people that would have previously resided in LTC facilities $[4,5]$. However, little is known in NZ, or elsewhere, about health or functional needs of this potentially vulnerable group of older people, or of what services are or are not provided by RVs to their residents.

In 2012 we completed a small feasibility survey of the functional status, dependency \& social circumstances of 110 participants in Auckland RVs [12]. Forty-five percent had been assessed for publicly funded homecare help while 15\% received help with personal cares. They had higher morbidity and need (vs. community-dwelling older people), including falls, general health, help with bathing/ dressing, and cognition. These findings suggest that physical function of RV residents lies between those living in private homes and those in LTC.

The basis for the current study was our belief that RV residents may have multiple unmet needs together with declining medical and functional 'trajectories' with time, and that a targeted intervention might decrease the risk first acute hospitalisation, number of all acute hospitalisations, LTC admissions and all-cause mortality.

The current study draws on the information gathered from residents themselves, from their healthcare records and at a facility level, to gain an overall better understanding of residents' health status and of RV service 
provision. It will inform understanding of characteristics, functional abilities, medical acuity and 'trajectories' (actuality and time scales of hospitalisation, move to LTC, mortality) of RV residents. This will, in turn, inform health planning to improve health outcomes in residents. The aims of the current paper are to provide an overall description of the overarching project and detailed methods information.

\section{Methods/design \\ Objectives}

We hypothesize RV residents have multiple unmet needs and high healthcare use and that a targeted intervention will decrease the risk of first acute hospitalisation, number of all acute hospitalisations, LTC admissions and allcause mortality.

The objectives of the study are:

1. To describe general health and functional status of residents;

2. To describe the types of facilities and services that RVs provide;

3. To examine the healthcare service utilization trajectories of RV residents over a three-year period (or longer depending on further funding);

4. To assess in a randomised controlled trial (RCT) the effect of a multidisciplinary team (MDT) intervention on healthcare outcomes in an identified, at-risk subgroup of RV residents.

\section{Study design}

This is a three-year project with data collection commencing in July 2016. This study comprises three phases: Survey phase - a description of RVs and of residents' characteristics and health and functional status; Cohort study phase - modeling of residents' health care trajectories up to 3 years (and longer if further funding can be secured); An RCT of a MDT intervention in the most vulnerable cohort of residents, aiming to reduce adverse outcomes. The study was approved by the New Zealand Health and Disability Ethics Committee (Ref 16/CEN/34).

\section{Setting}

A list of all RVs in the Auckland (ADHB) and Waitematā (WDHB) District Health Boards (that are responsible for providing and/or funding the provision of health services in their defined geographical area) was compiled from a variety of sources (online, previous study data and personal contacts). This list was used as the sampling framework for the recruitment of RVs and we planned that all RVs would be invited to take part regardless of size, ownership or location.

\section{Recruitment procedures}

We planned to approach all RVs in ADHB and WDHB, with random sampling of units/apartments in each village using the villages' own lists as the sampling frame. Exclusions: refusal of, or inability to consent (Addenbrookes Cognitive Examination Revised ACE-R) [13] under 65 , or any resident that our research gerontology nurse specialists (GNSs: AT and CC) or the resident's RV manager felt may lack capacity to consent. Ethics approval did not require written consent from the facility.

\section{Village managers}

A GNS allocated to each DHB contacted individual RV managers and gained verbal or email agreement for them to participate in a Village Management Survey. The purpose of this survey is to describe the RVs in the region, in terms of size, facilities and services provided, in order to give a detailed overview of the physical environment: the number of independent-living units or apartments, and 'serviced apartments' (units whose residents were able to access from the RV, at additional cost to themselves or to their DHB, services ranging from household help to personal care), recreational facilities accessible to residents, and healthcare services on site.

Where necessary, the Principal Investigator (MJC) contacted the Chief Executive Officers (CEOs) of large RV chains to explain the study and seek permission and access to the RV to improve the participation rate of RV managers and residents.

\section{Village residents}

Survey phase: We planned to access residents via RV managers, and to contact residents by letter-drop and door-knock. In smaller RVs ( $n \leq 60$ units/apartments), we planned to invite all residents to assess eligibility, whilst in larger RVs we planned to invite residents in a random sample of units (aiming for about 30 residents per RV).

Potential participants were approached using a variety of methods including letter-drop, RV newsletter, noticeboards, and resident meetings and, in larger villages, lists of units from village RV managers for random selection.

RCT resident recruitment: We planned to utilise the InterRAI Community Health Assessment (CHA) (https://www.interrai.org/community-health.html) [14] as a component of the assessment of residents in the Survey phase and to identify residents eligible for the RCT based on these data $[15,16]$.

As the study evolved and progressed, recruitment issues and other practical problems necessitated changes in the originally designed and funded protocol. These pragmatic changes are detailed in Table 1. Problems and issues specific to subject recruitment will form the subject of a separate publication. 
Table 1 Summary of planned and actual study design and conduct

\begin{tabular}{|c|c|c|}
\hline & Planned /expected & Implemented /actual \\
\hline \multicolumn{3}{|l|}{ Survey Phase: } \\
\hline Village sampling frame & $\begin{array}{l}\text { Sampling frame would be complete } \\
\text { except for new villages opening during } \\
\text { the study period and additional units } \\
\text { opening in existing villages. }\end{array}$ & $\begin{array}{l}\text { Some villages duplicated in list; and some } \\
\text { villages listed were out of area. } \\
\text { Villages closed units for renovation, or added } \\
\text { units/apartments } \\
\text { Facilities were listed that were not retirement } \\
\text { villages. } \\
\text { The details were updated during the course of } \\
\text { the Survey phase. }\end{array}$ \\
\hline Village selection & $\begin{array}{l}\text { No selection of villages was planned. All } \\
\text { villages would be approached, and we } \\
\text { expected any decline in participation to } \\
\text { be minor. }\end{array}$ & $\begin{array}{l}\text { Slower than anticipated study progress led to } \\
\text { recognition that not all villages could be included, } \\
\text { A change in policy saw villages being recruited in } \\
\text { random order after Dec } 2017 \text {. }\end{array}$ \\
\hline Village recruitment & $\begin{array}{l}\text { Gate-keeping role of village managers was } \\
\text { recognised, but the extent research team } \\
\text { were not given permission to recruit at all } \\
\text { was not anticipated. }\end{array}$ & $\begin{array}{l}\text { A high proportion of managers declined } \\
\text { participation early in study. This will be detailed } \\
\text { in a separate paper. }\end{array}$ \\
\hline Unit sampling frame & $\begin{array}{l}\text { Within each village we anticipated that a list } \\
\text { of all units/apartments (without names of } \\
\text { residents) would be provided by village } \\
\text { manager, enabling random selection of units, } \\
\text { together with permission and security access } \\
\text { for door-knocks and letter-drops allowing } \\
\text { recruitment to proceed. }\end{array}$ & $\begin{array}{l}\text { Some Managers declined to provide a list of units } \\
\text { and also to permit access to doors, making door- } \\
\text { knocks or even letter-drops impossible. Instead, these } \\
\text { managers permitted study nurses to present an outline } \\
\text { of the study at a residents' meeting, and seek volunteers } \\
\text { for participation. This will be detailed in a separate paper } \\
\text { as above. }\end{array}$ \\
\hline
\end{tabular}

Call-backs

Resident eligibility criteria

Resident sampling

Informed consent

Survey instrument(s)

Timeline

Sample size and Power calculations

Data availability and collection
Where at door-knock there was no response, the researchers were to call back up to 5 times, in accordance with Good Practice in Conduct and Reporting of Survey Research [17]. to avoid bias towards those who are frequently at home.

All residents in randomly-selected units would be invited to participate. Further, every resident under an LTC contract (i.e. receiving formal publicly-funded LTC services) would be included.

Where more than one resident was in a unit/ apartment, and both met resident inclusion criteria, then both were to be invited, to enable assessment of support from coresidence and inter-dependence.

All residents were eligible to participate regardless of health or occupancy status. For those without capacity to consent but with a legal proxy available, we planned to use the combination of respondent and proxy.

Both feasibility and pilot studies used questionnaires based on our prior research in residential care facilities, based on Booth questionnaire, BRIGHT study and ARCHUS tools.

We planned to begin the Survey phase recruitment on 06June 2016 and complete it by August 2018.

Refer to text.

Survey data were expected to be collected online and immediately available for use in monitoring progress, conducting follow-up and to determine eligibility for the RCT.
After several months of fieldwork, 10 call-backs was deemed too high a number for the yield provided, and call-backs were reduced to 3 .

All residents in each randomly-selected unit/apartment were invited. In villages where random selection was not undertaken, all residents were invited.

Few residents were in receipt of LTC contracts, and the numbers did not justify continuing this line of enquiry.

This went to plan.

On the basis of the NZ legislation, the ethics committee approval for the study did not permit recruitment of residents who did not have capacity to consent. This will be detailed in a separate paper as above.

Mandatory introduction of the interRAl tool nationally was deemed more relevant, appropriate and thorough than our instruments, so the interRAI CHA was added. We selected the interRAI CHA tool (previously unused in NZ) as our main study instrument, and adapted the trialled survey tool as a supplementary survey questionnaire to capture additional items such as reasons for moving into a RV.

We began Survey phase recruitment on 27 July 2016 and completed in late August 2018.

Refer to text.

Once the decision was made to use an interRAl instrument (where data are captured online and available only occasionally to researchers), it was impossible to use interRAl data directly for determining eligibility for Phase 3. Selected variables were therefore entered into the survey database for this purpose. 
Table 1 Summary of planned and actual study design and conduct (Continued)

\begin{tabular}{|c|c|c|}
\hline & Planned /expected & Implemented /actual \\
\hline \multicolumn{3}{|l|}{ Cohort Follow-up: } \\
\hline Timeline & From June 2016 to August 2018 & From July 2016 to January 2020 \\
\hline Length of follow-up & Average 1 year & Average 2.5 years \\
\hline Endpoints of interest & $\begin{array}{l}\text { Primary endpoint: First acute hospitalisations, } \\
\text { Secondary endpoints: ED presentation, LTC } \\
\text { admissions, mortality }\end{array}$ & $\begin{array}{l}\text { Primary endpoint: First acute hospitalisations, } \\
\text { Secondary endpoints: number of all acute } \\
\text { hospitalisations, LTC admissions, all-cause } \\
\text { mortality }\end{array}$ \\
\hline Power calculations & Refer to text & Refer to text \\
\hline \multicolumn{3}{|l|}{ RCT } \\
\hline Eligibility criteria & $\begin{array}{l}\text { Initially it was planned to recruit those from } \\
\text { the Survey phase sample deemed most } \\
\text { 'vulnerable' (to adverse outcomes) based on } \\
\text { selection criteria employed in our ARCHUS } \\
\text { study, including the number of medications } \\
\text { prescribed. }\end{array}$ & $\begin{array}{l}\text { Once the interRAl tool was chosen, interRAI based } \\
\text { criteria were used to select residents, including } \\
\text { triggering of } 3 \text { or more CAPS, (LTC institutionalisation } \\
\text { risk) scores of } 3 \text { or more or at the discretion of the } \\
\text { GNS. The number of medications reported in the } \\
\text { interRAI tool included over-the-counter medications. } \\
\text { On investigation, very few residents were being missed } \\
\text { if number of medication criteria was dropped, so that } \\
\text { inclusion criterion was removed. We retained from the } \\
\text { ARCHUS study the validated criterion of the GNS's } \\
\text { impression of vulnerability/medical acuity. }\end{array}$ \\
\hline Pre-randomisation checks & $\begin{array}{l}\text { All eligible for the trial were to be checked } \\
\text { by a study nurse before randomisation to } \\
\text { ensure those no longer eligible for the trial } \\
\text { were excluded from randomisation. }\end{array}$ & $\begin{array}{l}\text { Survey phase recruitment issues meant that resources } \\
\text { to conduct pre-randomisation checks were limited to } \\
\text { database checks and short contact with residents in } \\
\text { the active arm only, i.e. after randomisation. }\end{array}$ \\
\hline Sample size & Refer to text & Refer to text \\
\hline Timeline & $\begin{array}{l}\text { We originally planned to complete the RCT } \\
\text { in all villages by } 28 \text { Feb } 2019\end{array}$ & $\begin{array}{l}\text { We completed RCT randomisation for in mid-January } \\
2019 \text { and completed the final MDT on } 14 \text { March } 2019 .\end{array}$ \\
\hline $\begin{array}{l}\text { Interval between survey and } \\
\text { randomisation }\end{array}$ & $\begin{array}{l}\text { The interval between survey (and interRAl } \\
\text { assessment) was anticipated as being } \\
3 \text { months, so the assessment would } \\
\text { reasonably represent health status at time } \\
\text { of randomisation. }\end{array}$ & $\begin{array}{l}\text { An average interval of } 9 \text { months between survey and } \\
\text { randomisation was achieved. Consequently, some } \\
\text { assessments will misrepresent health status at time } \\
\text { of randomisation. }\end{array}$ \\
\hline Length of follow-up & $\begin{array}{l}\text { Planned follow up possibly at } 1,2 \& 4 \text { years } \\
\text { post-intervention. }\end{array}$ & $\begin{array}{l}\text { Planned follow up } 1.5 \text { years post intervention } \\
\text { MoH data. The } 4 \text { th years post-intervention } \\
\text { follow-up is conditional on obtaining additional } \\
\text { funding }\end{array}$ \\
\hline Endpoints of interest & $\begin{array}{l}\text { Primary endpoint: First cute hospitalisation } \\
\text { Secondary endpoints; All acute hospitalisations, } \\
\text { LTC admissions, mortality }\end{array}$ & $\begin{array}{l}\text { Primary endpoint: First acute hospitalisation } \\
\text { Secondary endpoints: number of all acute } \\
\text { hospitalisation, LTC admissions, mortality } \\
\text { Power analyses are based on the primary outcome. } \\
\text { We did consider changing the primary outcome to } \\
\text { ED presentations, but decided against this as, in NZ, } \\
\text { many people who are acutely hospitalised are not } \\
\text { admitted via ED but go via GP referral to acute } \\
\text { admitting units. }\end{array}$ \\
\hline Power calculations & $\begin{array}{l}\text { That residents were clustered into villages } \\
\text { led to power calculations including an } \\
\text { adjustment for a cluster effect. }\end{array}$ & $\begin{array}{l}\text { Clustering was catered for by stratified random } \\
\text { sampling, and adjustment for cluster effect was } \\
\text { unnecessary. }\end{array}$ \\
\hline
\end{tabular}

Survey baseline Recruited residents, after GNSs obtained their written, informed consent, were asked to complete a questionnaire facilitated by the GNSs that provided details of demographic, social engagement, and decision-making paradigms. Items in the resident survey included factors that influenced move to a $\mathrm{RV}$, previous home ownership, home-based support services currently receiving, satisfaction in aspects of RV living, types and extent of social and physical activities currently undertaken, use of dental and other health care services, internet usage, loneliness, spiritual wellbeing and any planned surgery or medical intervention. This questionnaire was informed by our previous feasibility study [12]. Recruitment began in July 2016 and was completed in August 2018.

Where a prior interRAI Home Care (HC) (https:// www.interrai.org/home-care.html) or Long Term Care Form (LTCF) (https://www.interrai.org/long-term-care- 
facilities.html) assessment had been completed within the past 6 months, this assessment was used. The purposes of an interRAI assessment is to accurately determine the characteristics of a person in order to fully describe their needs, ranging from clinical to social support. The outcome measures of the interRAI assessment are known as Clinical Assessment Protocols (CAPS) and are designed to assist the healthcare professional to interpret systematically all the information recorded [14].

We also utilised InterRAI-CHA as a component of assessment in the Survey phase, and for selection for the RCT. "the InterRAI CHA Assessment form is a Minimum Data Set (MDS) screening tool that enables an assessor to review multiple key domains of function, health social support and service use. Particular interRAI CHA items also identify persons who could benefit from further evaluation of specific problems or risks for functional decline. These items, known as triggers, link the interRAI CHA to the problem orientated CAPs. The CAPs provide general guidelines for further assessment and individualised care and services".

Cohort study follow-up Study outcomes will be to describe trajectories of healthcare utilisation. The primary outcome will be first acute hospitalisation, while secondary outcomes will number of all acute hospitalisations, LTC admissions and all-cause mortality. All are available from routinely collected Ministry of Health $(\mathrm{MOH})$ administrative data, by use of each individual's unique $\mathrm{Na}$ tional Health Identifier (NHI). For any subjects declining entry into the RCT (below), GNSs confirmed their continued permission to obtain these centrally-held data.

RCT Based on a combination of the interRAI assessment and selection criteria validated from our ARCHUS study [15] a sub-sample of resident from the baseline phase (initially estimated at about 28\%) 'at high risk' of health and functional decline, will be selected for inclusion in the RCT (Table 1). These potentially 'at risk' residents, eligible for the RCT, will be identified by triggering three or more interRAI CAPS. There will be no additional exclusion criteria applied. After GNSs obtained written, informed consent, eligible residents will then be randomly allocated to the control or intervention arm, stratified by village. The randomisation list will be generated (using computer-based random number generation) by a statistician not involved in outcome measures. This will ensure that the two groups are balanced regarding each RV. The controls will receive no additional contact, assessments or services. The intervention group will receive a GNS-facilitated intervention plan, similar to our ARCHUS study [15]. In brief, this comprises a comprehensive geriatric assessment by a clinical team consisting of a GNS, a geriatrician and a clinical pharmacist reviewing baseline 1 assessments, residents' medical records and a further update of health issues prior to the MDT meeting. If an urgent significant health issue is identified, intervention subjects will receive a direct referral from the research team to other relevant services and/or a phone call to the GP. Randomisation and MDTs began in March 2017.

The MDT review will occur in collaboration with the older person \& their chosen support person(s), geriatrician or nurse practitioner, and clinical pharmacist. This will culminate in an MDT meeting (usually in the resident's apartment) lasting approximately $45 \mathrm{~min}$, to which the subjects' general practitioners (GPs) will be invited. Treatment goals and recommendations will be discussed and developed with the resident and all present, and a summary of the MDT meeting with suggestions and recommendations sent by mail to each participant and their GP.

The effect of the intervention on health outcomes will be assessed by this RCT. Healthcare use (NZ Ministry of Health databases) using the resident's unique NHI number will be evaluated 1-year pre and 1, 2- \& 4-years post-intervention (the latter depending on further research funding being obtained). The Primary Outcome will be first acute hospitalisation. Secondary outcomes will comprise the number of all acute hospitalisations, LTC admissions, and all-cause mortality.

\section{Data management}

The two surveys used online Qualtrics tool (https:// www.qualtrics.com). InterRAI assessments are also conducted online, using the same systems as all NZ needs assessments are undertaken. Electronic lists of the unique study identification numbers, date of consent to the study and NHI number will be sent to the interRAI organization and $\mathrm{MoH}$ for data extract purposes. Outcome and health service use data will then be provided by interRAI and the $\mathrm{MoH}$ by secure link to the researchers, identified by study ID, i.e. without NHI numbers, names or any other identifiers. Data are stored on password-protected shared drives available only to bona fide members of the university research team, and located in locked University offices.

Similarly, health outcomes data for the Survey phase and the RCT will be obtained from $\mathrm{MoH}$ from an electronic file supplied by the research team, following an approval process. Data from the two surveys, from interRAI and from $\mathrm{MoH}$ will be imported into SAS version 9.4 (SAS Institute Inc., Cary. NC, USA) used for all analyses.

\section{Power and sample size calculation}

Initial power calculation for the RCT was performed prior to commencement of the Survey phase. Following 
commencement of the Survey phase it became apparent that the subjects were of higher medical acuity and vulnerability than we had anticipated and revised power calculations for the RCT were performed before RCT commencement.

Sample size for the Survey phase follow-up phases was largely governed by the sample needed for the RCT. An estimated $65 \%$ of the Survey phase follow-up residents were eligible for the RCT, meaning that 572 residents were needed in the Survey phase. An average of 2.5 years follow-up will be available for the Cohort phase by January 2020. If the rate of acute hospitalisation (primary outcome) is $55 \%$ per year we will have at least $80 \%$ power to show a $20 \%$ difference in the hazard ratio for any two categories.

For the RCT, we estimated 372 residents met the "high-risk" criteria, will be randomised and followed for 1 year after randomisation. The rate of acute hospitalisation in LTC residents is $67 \%$ per year (from previous work). We conservatively hypothesised the acute hospitalisation rate in the "high risk" RCT RV residents would be $70 \%$ per person-year. We would expect $80 \%$ power to show a $20 \%$ reduction in our primary outcome (acute hospitalisation). If the effect is stronger (e.g. $25 \%$ or $30 \%$ - as found in our ARCHUS [16] and ARCHIP [18] studies or longer follow-up, power will increase, though based on our earlier work (ARCHIP), the duration of any intervention effect may be under 1.4 years.

\section{Statistical analysis}

In the Survey phase, initial descriptive analyses will be conducted. Then, in both the Survey and Cohort phases, multivariate regression models (including logistic regression, log-binomial regression, linear regression and Cox regression) will be used to explore the influence factors on health outcomes adjusting for potential confounders (demographics, lifestyle, medical history, clinical characteristics, village characteristics etc.) and time to event when possible.

For the primary and main secondary outcomes in the RCT, analyses will be conducted on an intentionto-treat basis. Cox proportional regression models, with robust sandwich variance estimates, will be used to compare the time to health outcomes between the intervention and usual care groups. Hazard ratios (HRs) and 95\% confidence intervals (CIs) will be calculated. Sub-group analyses will evaluate treatment effect for the main endpoint for different sub-groups, e.g. subgroup analyses for each endpoint by sex, large/small village, clinic/nurse/not, sampled/volunteers. Analyses will be conducted by a research statistician not involved in the clinical aspects of the study.

\section{Study status}

Recruitment to the Survey phase ended in August 2018. RCT randomisation was completed in mid-January 2019 and all MDTs were completed by 14 March 2019. This will give us, by end of January 2020, a median (range) 29 (18-42) months of Cohort follow-up and 18 (12-33) months RCT follow-up. Trial results will be the subject of further publications and communicated to residents by regular newsletters.

\section{Discussion}

The aims of the Older People in Retirement Villages project are to describe RVs within the study region and the health and functional status of residents (Survey phase); to examine these over a two-to-four-year period (Cohort follow-up); and to assess the effect of a MDT intervention in an identified at risk group of residents (RCT) on the healthcare outcomes of first acute hospitalisation, all acute hospitalisations, LTC admission and all-cause mortality. It is the largest cross-sectional study of RVs in NZ and has enabled a longitudinal cohort study of healthcare utilisation and a randomised controlled trial of comprehensive gerontology intervention. Its potential weakness relates to difficulties in recruitment of both retirement villages and of village residents, potentially affecting representativeness of recruited subjects and summarized in Table 1. These issues will form the basis of a separate paper.

The findings from this project will represent the first comprehensive portrayal of older RV residents $\mathrm{NZ}$ and an overview of RV settings. The results from this study will help inform policy development and health care provision strategies, as it will provide a clearer picture of unmet need and the effect of a targeted intervention in this population. This trial will be of interest to service users (residents and potential residents) illustrating how social and physical environmental settings are characterized, and possibly how the older population are ageing in RVs compared to other conglomerate housing settings.

In addition, social connection and participation are considered an aspect of RV living that flourishes [19]. A recent NZ study involving $163 \mathrm{RV}$ residents in two RVs found that a "dignified" environment improved mental wellbeing, provided an environment that built relationships and reduced loneliness and isolation [11]. However, some international studies have shown that family contact reduces for village residents compared to community dwelling older people. Given the large and increasing numbers of older New Zealanders living in RVs, it is surprising and potentially concerning that we know so little about them. The current study will further explore these areas and its 
results could have wider ranging implications for future service planning for RV residents, including but not restricted to those who develop complex physical or cognitive needs. RVs are not unique to $\mathrm{NZ}$ and our findings will inform planning and provision internationally.

Our previous work in the LTC sector indicates that targeting of vulnerable, co-morbid older people and offering GNS-led, complex intervention reduces unnecessary hospitalisations for many conditions by over $20 \%$ (vs. usual care) [16, 18]. It is feasible that such interventions (as in the current study's RCT) will similarly benefit RV residents.

We anticipate that our findings and experiences will also provide insights into the opportunities and challenges of collaboration with RVs and provide recommendations to future research partnerships.

\section{Abbreviations}

RVs: Retirement villages; NZ: New Zealand; MDT: Multidisciplinary team; GNS: Gerontology nurse specialist; RCT: Randomised controlled trial; CCRC: Continuing care retirement community; LTC: Long-term care; ED: Emergency department; ACE-R: Addenbrookes cognitive examination revised; CHA: Community health assessment; HC: Home care; LTCF: Long term care form; CAPS: Clinical assessment protocols; MDS: Minimum data set; CAPs: Clinical assessment protocols; $\mathrm{MOH}$ : Ministry of health; $\mathrm{NHI}$ : National health Identifier; GPs: General practitioners; HRs: Hazard ratios; Cls: Confidence intervals

\section{Acknowledgements}

We are enormously grateful to all retirement village managers and village residents who participated in this trial, to the Retirement Village Association of New Zealand, and to General Practitioners in the Auckland and Waitemata districts.

We thank Gabrielle Peters for her assistance with manuscript preparation, and administration tasks of the research project.

We are especially grateful to Marilyn Crawley, Chief Pharmacist, Pharmacy Department, Waitemata District Health Board, for her valued assistance in organising the support of Clinical Pharmacists to this project.

\section{Authors' contributions}

KP drafted, edited feedback and finalised manuscript writing; MJC obtained funding for the study and led design. $\mathrm{KP}, \mathrm{KB}, \mathrm{MB}, \mathrm{JBB}$ and $\mathrm{DB}$ also contributed to study design and funding application. MJC, KP, KB, MB, AT, $\mathrm{CC}$, and $\mathrm{JH}$ were the clinical investigators. A-MH led on study logistics including logistics of subject-investigator meetings. ZW and JBB led the statistical analyses. JBB developed summary of planned and actual study design table. All authors (KP, JBB, JH, MB, KB, ZW, CC, AT, A-MH, DB, and MJC) contributed to reading, editing and approving the final manuscript. We did not use professional manuscript writers.

\section{Funding}

The study was funded by a grant from New Zealand's National Science Challenge - Ageing Well ('Project F' - UOOX1508, 12815/1, SUB1301), and from Waitematā District Health Board (WDHB). The funders and sponsor had no input into the design, execution or reporting of the study. Sponsor: University of Auckland, School of Medicine. Professor Phillippa Poole.p.poole@auckland.ac.nz

\section{Availability of data and materials}

Not applicable for this paper because no data has been generated. Participants are and will be updated regarding study results by newsletter.

\section{Ethics approval and consent to participate}

This study was approved by the New Zealand Health and Disability Ethics Committee (Ref 16/CEN/34). All participants provided written, informed consent.

\section{Consent for publication}

Not applicable.

\section{Competing interests}

The authors declare that they have no conflicts of interest.

\section{Author details}

${ }^{1}$ School of Nursing, University of Auckland, Private Bag 92 019, Auckland 1142, New Zealand. ${ }^{2}$ Department of Geriatric Medicine, University of Auckland, Level 1, Building 5, Waitemata District Health Board, PO Box 93 503, Auckland, Takapuna 0740, New Zealand. ${ }^{3}$ Waitemata District Health Board, PO Box 93 503, Auckland, Takapuna 0740, New Zealand. ${ }^{4}$ Auckland District Health Board, Private Bag 92 024, Auckland Mail Centre, Auckland 1142, New Zealand.

Received: 9 June 2019 Accepted: 3 July 2020

Published online: 17 July 2020

\section{References}

1. Petersen M, Tilse C, Cockburn T. Living in a Retirement Village: choice, contracts, and constraints. J Hous Elder. 2017;31(3):229-42.

2. Retirement Villages Act and Regulations. Wellington: New Zealand Ministry of Housing and Urban Development; 2003. https://www.hud.govt.nz/ residential-housing/retirement-villages/retirement-villages-act-andregulations/.

3. Broad JB, Boyd M, Kerse N, Whitehead N, Chelimo C, Lay-Yee R, von Randow M, Foster S, Connolly MJ. Residential aged care in Auckland, New Zealand 1988-2008: do real trends over time match predictions? Age Ageing. 2011;40(4):487-94.

4. New Zealand Positive Ageing Strategy. Wellington: New Zealand Ministry of Social Development; 2001. https://www.msd.govt.nz/aboutmsd-and-ourwork/publications-resources/planning-strategy/positive-ageing/.

5. Caring for Frail Elderly People: New Directions in Care. Paris: Organisation for Economic Cooperation and Development; 1994.

6. Boyd M, Broad JB, Kerse N, Foster S, von Randow M, Lay-Yee R, Boyd M, Broad JB, Kerse N, Foster S, von Randow M, Lay-Yee R, Chelimo C, Whitehead N, Connolly MJ. Twenty year trends in dependency in residential aged Care in Auckland, New Zealand: a descriptive study. J Am Med Dir Assoc. 2011:12:535-40.

7. Boyd M, Broad JB, Kerse N, Foster S, von Randow M, Lay-Yee R, Chelimo C, Whitehead N, Connolly MJ. Twenty-year trends in dependency in residential aged care in Auckland, New Zealand: a descriptive study. J Am Med Dir Assoc. 2011:12(7):535-40.

8. LaSalle LJ: New Zealand Retirement Village database (NZRVD) November 2017. Whitepaper February 2018.

9. Kennedy DJ, Coates D. Retirement Village resident satisfaction in Australia: a qualitative enquiry. J Hous Elder. 2008;22(4):311-34.

10. Gardner IL, Browning C, Kendig H. Accommodation options in later life: retirement village or community living? Australas J Ageing. 2005;24(4):18895.

11. Yeung P, Good G, O'Donoghue K, Spence S, Ros B. What matters most to people in retirement villages and their transition to residential aged care. Aotearoa New Zealand Social Work. 2017;29:84-96.

12. Bhandal P, Siew L, Broad JB, Boyd M. Feasibility study to describe the dependency levels of people living in retirement villages in Auckland, New Zealand. West J Nurs Res. 2012;31(4):462-79.

13. Mathuranath PS, Nestor PJ, Berrios GE, Rakowicz W, Hodges JR. A brief cognitive test battery to differentiate Alzheimer's disease and frontotemporal dementia. Neurology. 2000;55(11):1613-20.

14. Morris J, Berg K, Björkgren M, Declercq A, Finne-Soveri H, Fries B, Frijters DF, Gilgen R, Gray L, Henrard J, Hirdes J, Ljunggren G, Nonemaker S, Steel K, Szcerbińshla K. interRAl Community Health (CHA) Assessment Form and User's Manual and Related Materials. Washington DC: interRAl; 2010.

15. Connolly M, Boyd M, Broad J, Kerse N, Lumley T, Whitehead N, Foster S. The Aged Residential Care Healthcare Utilisation Study (ARCHUS): a 
multidisciplinary, cluster-randomised, controlled trial designed to reduce avoidable hospitilisations from long-term care facilities. J Am Med Dir Assoc. 2015;16(2015b):49-55.

16. Connolly MJ, Broad JB, Boyd M, Zhang TX, Kerse N, Foster S, Lumley T, Whitehead N. The 'Big Five'. Hypothesis generation: a multidisciplinary intervention package reduces disease-specific hospitalisations from longterm care: a post hoc analysis of the ARCHUS cluster-randomised controlled trial. Age Ageing. 2016;4:415-20.

17. Kelley K, Clark B, Brown V, Sitzia J. Good practice in the conduct and reporting of survey research. Int J Qual Health Care. 2003;15:261-6.

18. Connolly MJ, Broad JB, Bish T, Zhang X, Bramley D, Kerse N, Bloomfield K, Boyd M. Reducing emergency presentations from long-term care: a beforeand-after study of a multidisciplinary team intervention. Maturutas. 2018: 117:45-50.

19. Buys L. Life in a retirement village: implications for contact with the community and village friends. Gerontology. 2001;47:55-9.

\section{Publisher's Note}

Springer Nature remains neutral with regard to jurisdictional claims in published maps and institutional affiliations.

Ready to submit your research? Choose BMC and benefit from:

- fast, convenient online submission

- thorough peer review by experienced researchers in your field

- rapid publication on acceptance

- support for research data, including large and complex data types

- gold Open Access which fosters wider collaboration and increased citations

- maximum visibility for your research: over $100 \mathrm{M}$ website views per year

At BMC, research is always in progress.

Learn more biomedcentral.com/submissions 